\title{
RELAÇÕES ENTRE PRECIPITAÇÕES INTENSAS DE DIFERENTES DURAÇÕES DE VIDEIRA, SANTA CATARINA
}

\section{RELATIONSHIPS BETWEEN HEAVY PRECIPITATIONS OF DIFFERENT DURATIONS OF VIDEIRA, SANTA CATARINA STATE, SOUTH OF BRAZIL}

\begin{abstract}
Álvaro José Back ${ }^{1}$ Augusto Carlos Pola ${ }^{2}$

Resumo

Este artigo apresenta a atualização das relações Intensidade-Duração-Frequência de chuvas para o município de Videira, SC. Foram utilizados os dados cotados dos pluviogramas da Estação Meteorológica do Instituto Nacional de Meteorologia (INMET) localizada em Videira, SC (latitude $27^{\circ} 00^{\prime} \mathrm{S}$, longitude $51^{\circ} 09^{\prime} \mathrm{W}$ ) do período de 1986 a 2014. Com base nas séries de máximas anuais de precipitação com duração de 5 até 1440 minutos, e utilizando a distribuição de Gumbel, foram estimadas as intensidades máximas de chuva com período de retorno de 2 a 100 anos. Foram ajustadas duas equações para a estimativa das intensidades das chuvas, uma para durações de até 120 minutos, outra para durações de 120 a 1440 minutos. Também foram determinadas as relações entre chuvas de diferentes durações, sendo observada uma variação na relação entre a chuva máxima de 24 horas e a chuva máxima de um dia conforme a hora da coleta da chuva diária. Observou-se que as relações entre chuvas de diferentes durações de Videira apresentaram valores ligeiramente inferiores aos valores médios recomendados para uso no Brasil. A relação média da chuva de 24 horas em relação a chuva máxima de um dia foi de 1,14 .
\end{abstract}

Palavras chave: chuvas intensas, equação IDF, probabilidade.

\begin{abstract}
This article presents the upgrade of rainfall's intensity-duration-frequency relation for the city of Videira, SC. Pluviograms from the Weather Station of the National Institute of Meteorology (INMET) located in Videira, SC (latitude $27^{\circ} 00$ 'S, longitude $51^{\circ} 09^{\prime} \mathrm{W}$ ) were used from 1986 to 2014. Based on the maximum annual precipitation series with duration of 5 to 1440 minutes and using the Gumbel distribution, maximum intensity of rainfall with a return period of 2 to 100 years were estimated. Two equations were adjusted to estimate the intensity of rainfall, one for up to 120 minutes' length and other for between 120 and 1440 minutes' length. It was also determined the relationship between different lengths' rainfall, being observed a variation in the relationship between the maximum rain in 24 hours and the maximum rain in one day according to the time of the daily rain's measurement. It was observed that the relationship between rainfall of different length observed in Videira were slightly lower than the average values recommended for use in Brazil. The ratio between the average rain in 24 hours and the average rain in one day was 1,14 .
\end{abstract}

Keywords: heavy rainfall, IDF equation, probability.

\footnotetext{
${ }^{1}$ Engenheiro agrônomo, Dr. em Engenharia, pesquisador da Empresa de Pesquisa Agropecuária e Extensão Rural de Santa Catarina (Epagri), estação experimental de Urussanga, rodovia SC 108, km 1563, Caixa Postal 49, Urussanga, SC, CEP 88840-000, ajb@epagri.sc.gov.br

2 Engenheiro agrônomo, Mestre em Agrometeorologia, pesquisador da Empresa de Pesquisa Agropecuária e Extensão Rural de Santa Catarina (Epagri), pola@epagri.sc.gov.br
} 


\section{Introdução}

O conhecimento das relações entre as grandezas intensidade, duração e frequência (IDF) de chuvas intensas é de grande importância nos estudos hidrológicos e no dimensionamento de obras relacionadas à drenagem pluvial ou captação de água da chuva. Essas relações podem ser expressas de forma gráfica nas curvas IDF ou por meio das equações de chuvas intensas, que podem ser obtidas por meio da análise de longas séries de registros pluviográficos.

Quando não se dispõe de longas séries de informações pluviográficas, uma técnica alternativa muito empregada é a desagregação da chuva diária em intervalos de menor duração (TOMAZ, 2002; BACK, 2013). A desagregação baseia-se em relações observadas entre as chuvas intensas de diferentes durações. No Brasil essa técnica tem sido muito utilizada, sendo aplicada com base nas relações entre precipitações de diferentes durações apresentadas pela Cetesb (1986).

Segundo Genovez e Zuffo (2000), as relações entre precipitações de diferentes durações apresentadas pela Cetesb (1986), além de utilizarem períodos muito curtos, foram obtidas para uma média nacional. Esses valores médios nacionais deveriam ser revistos para os de médias regionais contemplando-se, dessa forma, as precipitações predominantes em cada região. Os autores destacam, também, que esses valores necessitam ser atualizados, por se tratar de um estudo feito há muito tempo, baseado nas curvas de intensidade de precipitação-duração-frequência para 98 localidades do Brasil, obtidas por Pfafstetter (1957), utilizando períodos de dados muito curtos sendo, para a maioria dos locais, da ordem de 10 anos de dados pluviográficos.

Em Santa Catarina existem poucas estações pluviográficas disponíveis para as estimativas das equações IDF. Back (2013) apresenta as equações IDF para treze estações pluviográficas, entres as quais a estação de Videira, com dados de 1986 a 2004. $\mathrm{Na}$ maioria destas estações a série de dados é inferior a 25 anos. Por outro lado, existem centenas de estações pluviométricas (ANA, 2009) em que muitas delas apresentam séries superiores a cinquenta anos de dados possibilitando a utilização da técnica de desagregar a chuva máxima diária, sendo uma alternativa muito útil nos estudos hidrológicos. Para aplicação dessa técnica é importante o conhecimento e a atualização das relações entre precipitações de diferentes durações para a região em estudo.

Este trabalho tem como objetivo atualizar as equações de chuvas intensas, e estabelecer as relações entre chuvas máximas de diferentes durações para o município de Videira, na região do Meio Oeste de Santa Catarina. 


\section{Material e métodos}

Foram analisados os pluviogramas da Estação Meteorológica de Videira, estado de Santa Catarina (latitude $27^{\circ} 00^{\prime} \mathrm{S}$, longitude $51^{\circ} 09^{\prime} \mathrm{W}$, altitude $774 \mathrm{~m}$ ), pertencente a Empresa de Pesquisa Agropecuária e Extensão Rural de Santa Catarina (Epagri), relativos ao período de 1986 a 2014. Os pluviogramas foram digitalizados e armazenados em meio magnético. Elaborou-se um programa para discretizar os arquivos digitalizados em intervalos de um minuto, e posteriormente determinar as séries de máximas anuais para as durações de 5 até 1440 minutos.

Foi utilizada a equação de Gumbel-Chow (CHOW, 1964) para estimar a chuva máxima com período de retorno de 2 a 100 anos. A aderência dos dados à distribuição ajustada foi avaliada com o teste de Kolmogorov-Smirnov a $5 \%$ de probabilidade (NEGHETTINI e PINTO, 2013).

A precipitação máxima esperada foi estimada pela distribuição de Gumbel-Chow (CHOW, 1964) segundo a equação:

$$
\mathrm{X}_{\mathrm{T}}=\beta+\frac{\mathrm{Y}}{\alpha}
$$

Em que: $X_{\mathrm{T}}$ é a estimativa da chuva máxima, $\alpha$ e $\beta$ são os parâmetros da distribuição de Gumbel, Y é a variável reduzida.

Os parâmetros da distribuição de Gumbel foram estimados por:

$$
\begin{aligned}
& \alpha=\frac{\mathrm{Sn}}{\sigma} \\
& \beta=\mu-\frac{\mathrm{Yn}}{\alpha}
\end{aligned}
$$

Em que: $\mu$ é a média dos valores observados de X; $\sigma$ é o desvio padrão dos valores observados de X; Yn e Sn são respectivamente a média e o desvio padrão (Back, 2013) da variável reduzida $\mathrm{Y}$, que é estimada por:

$$
Y=-\ln \left\{-\ln \left[1-\left(\frac{1}{T}\right)\right]\right\}
$$

Em que: T é período de retorno.

As relações intensidade-duração-frequência de chuvas foram representadas por equações do tipo:

$$
i=\frac{K T^{\mathrm{m}}}{(\mathrm{t}+\mathrm{b})^{\mathrm{n}}}
$$


Em que: i é a intensidade de chuva $(\mathrm{mm} / \mathrm{h})$; $\mathrm{T}$ é período de retorno (anos); $\mathrm{t}$ é a duração de chuva (minutos); K, m, b, n são parâmetros da equação que devem ser ajustados aos dados observados.

O ajuste dos parâmetros foi feito minimizando a soma dos quadrados dos desvios (S) para todas as durações e períodos de retorno considerado, segundo a expressão:

$$
\mathrm{S}=\sum_{\mathrm{d}=1}^{\mathrm{n}_{\mathrm{d}}} \sum_{\mathrm{T}=1}^{\mathrm{n}_{\mathrm{T}}}\left(\mathrm{fi}_{\mathrm{d}, \mathrm{T}}-\mathrm{fo}_{\mathrm{d}, \mathrm{T}}\right)^{2}
$$

Em que: $\mathrm{fi}_{\mathrm{d}, \mathrm{T}}$ é a intensidade estimada para a duração $\mathrm{d}$, e período de retorno $\mathrm{T} ;$ fo $_{\mathrm{d}, \mathrm{T}}$ é a intensidade observada para a duração $\mathrm{d}$, e período de retorno $\mathrm{T}, \mathrm{n}_{\mathrm{d}}$ é o número de durações utilizadas e $\mathrm{n}_{\mathrm{T}}$ é o número de períodos de retorno utilizados.

\section{Resultados e discussão}

Na Tabela 1 são apresentados as estatísticas dos valores observados para cada duração estudada e os parâmetros da distribuição de Gumbel ajustada. O coeficiente de variação, dado pela relação entre o desvio padrão e a média, variou entre 16,6\% para a duração 5 minutos, até 28,2\% para a duração de 120 minutos. As séries também mostraram leve assimetria positiva, com coeficiente de assimetria variando entre 0,26 para a duração de 420 minutos a 1,23 para a duração 1440 minutos. Os valores de desvio máximo entre as frequências observadas e teóricas $\left(D_{\max }\right)$, do teste de Kolmogorov-Smirnov, para todas as durações foram inferiores aos valores críticos ao nível de 5\% $\left(\mathrm{D}_{\text {crti }}=0,2500\right)$ indicando que a distribuição de Gumbel-Chow pode ser usada. $\mathrm{O}$ maior valor $\left(\mathrm{D}_{\max }=0,1237\right)$ foi observado para a série com duração de 24 horas, e somente quatro séries apesentaram $D_{\max }$ superior a 0,10 , mostrando a boa aderência dos dados às séries ajustadas. Estes resultados encontram respaldo em vários trabalhos já também constataram que a distribuição de Gumbel é indicada para a estimativa das chuvas máximas (CLARKE, 1994; CLARKE, 2002).

Na Tabela 2 constam as intensidades de chuvas estimadas com a distribuição de Gumbel ajustadas para cada duração, cujos parâmetros foram apresentados na Tabela 1. A equação de chuvas intensas ajustada para estimar a intensidade das chuvas com duração de até 120 minutos está representada na Figura 1, e a equação para estimar a intensidade da chuva com duração de 120 a 1440 minutos está representada na Figura 2. Para a primeira equação o erro padrão de estimativa é de $3,3 \mathrm{~mm} / \mathrm{h}$ e o coeficiente de determinação $\mathrm{R}^{2}$ é de 0,9928 e para a segunda equação o erro padrão de estimativa foi de $0,70 \mathrm{~mm} / \mathrm{h}$ e o coeficiente de determinação $\mathrm{R}^{2}$ foi 0,9954 , que demonstram a boa 
qualidade obtida no ajuste das equações. Estas equações são recomendadas para serem usadas na região de Videira, SC.

Tabela 1 - Estatísticas observadas e parâmetros da distribuição de Gumbel para as séries e máximas anuais de precipitação de Videira, SC.

\begin{tabular}{ccccccccc}
\hline \multirow{2}{*}{$\begin{array}{c}\text { Duração } \\
\text { (minutos) }\end{array}$} & \multicolumn{9}{c}{ Estatísticas (mm) } & \multicolumn{4}{c}{ Distribuição Gumbel-Chow } \\
\hline 5 & 10,4 & 1,74 & 14,6 & 7,8 & 0,36 & 0,6356 & 9,606 & 0,0877 \\
10 & 15,1 & 3,34 & 23,0 & 10,1 & 0,35 & 0,3306 & 13,522 & 0,1105 \\
15 & 19,1 & 4,62 & 30,4 & 10,8 & 0,31 & 0,2390 & 16,833 & 0,1272 \\
20 & 23,2 & 5,72 & 36,2 & 13,7 & 0,51 & 0,1930 & 20,403 & 0,0932 \\
25 & 25,8 & 6,27 & 40,4 & 16,1 & 0,47 & 0,1761 & 22,761 & 0,0957 \\
30 & 28,2 & 7,28 & 45,1 & 16,5 & 0,41 & 0,1517 & 24,714 & 0,0889 \\
35 & 30,3 & 8,02 & 48,7 & 18,2 & 0,61 & 0,1378 & 26,404 & 0,0641 \\
40 & 32,1 & 8,12 & 51,6 & 20,1 & 0,70 & 0,1360 & 28,185 & 0,0679 \\
45 & 33,8 & 8,27 & 53,8 & 22,1 & 0,74 & 0,1335 & 29,781 & 0,0637 \\
50 & 35,4 & 8,37 & 54,3 & 24,1 & 0,68 & 0,1320 & 31,365 & 0,0783 \\
55 & 36,6 & 8,70 & 54,6 & 25,0 & 0,56 & 0,1269 & 32,404 & 0,0599 \\
60 & 37,5 & 9,00 & 54,6 & 25,2 & 0,48 & 0,1228 & 33,117 & 0,0595 \\
75 & 40,0 & 10,11 & 63,2 & 25,9 & 0,57 & 0,1093 & 35,127 & 0,0520 \\
90 & 42,2 & 11,45 & 72,6 & 26,7 & 0,81 & 0,0965 & 36,638 & 0,0526 \\
105 & 44,4 & 12,26 & 75,4 & 27,6 & 0,96 & 0,0901 & 38,485 & 0,0644 \\
120 & 46,4 & 13,11 & 78,0 & 27,7 & 0,87 & 0,0843 & 40,083 & 0,0772 \\
150 & 49,1 & 13,80 & 81,1 & 30,4 & 0,70 & 0,0801 & 42,424 & 0,0805 \\
180 & 51,7 & 13,71 & 81,3 & 33,4 & 0,52 & 0,0806 & 45,068 & 0,0947 \\
240 & 55,6 & 13,91 & 85,3 & 37,8 & 0,48 & 0,0794 & 48,857 & 0,0916 \\
300 & 58,9 & 14,68 & 92,9 & 38,7 & 0,48 & 0,0753 & 51,770 & 0,1111 \\
360 & 61,9 & 15,14 & 93,4 & 38,7 & 0,27 & 0,0730 & 54,587 & 0,0737 \\
420 & 64,7 & 15,45 & 93,6 & 40,0 & 0,26 & 0,0715 & 57,178 & 0,0646 \\
480 & 66,9 & 15,52 & 100,4 & 44,0 & 0,45 & 0,0712 & 59,402 & 0,0875 \\
600 & 70,5 & 14,71 & 100,4 & 48,9 & 0,41 & 0,0751 & 63,374 & 0,0780 \\
720 & 76,7 & 16,54 & 116,8 & 55,3 & 0,72 & 0,0668 & 68,724 & 0,0879 \\
840 & 80,6 & 17,68 & 124,9 & 56,3 & 0,74 & 0,0625 & 72,079 & 0,0565 \\
960 & 84,6 & 19,54 & 137,6 & 56,3 & 0,74 & 0,0565 & 75,108 & 0,0592 \\
1080 & 87,4 & 21,13 & 145,4 & 56,3 & 0,86 & 0,0523 & 77,201 & 0,0965 \\
1200 & 89,9 & 22,81 & 155,7 & 56,7 & 1,11 & 0,0484 & 78,890 & 0,0990 \\
1320 & 92,5 & 23,83 & 163,2 & 56,8 & 1,21 & 0,0463 & 80,906 & 0,0991 \\
1440 & 93,5 & 24,38 & 165,8 & 56,8 & 1,23 & 0,0453 & 81,682 & 0,1237 \\
\hline
\end{tabular}

${ }^{1} \mathrm{DP}=$ Desvio padrão. ${ }^{2} \mathrm{CA}=$ coeficiente de Assimetria

A relação entre a precipitação máxima em 24 horas e a precipitação máxima e um dia normalmente utilizada varia entre 1,10 e 1,14 (BERTONI e TUCCI, 1993, TOMAZ, 2002). Occhipinti e Santos (1966) utilizando séries de dados de 1928 a 1965 de São Paulo, observaram a relação variando de 1,13 a 1,15 com média de 1,14. Genovez e Pegoraro (1998) obtiveram, analisando dados de 23 pluviógrafos do Estado 
de São Paulo, valor mínimo de 1,03 e máximo de 1,17, com média de 1,13. Torrico (1975) propõe, baseado nas estações usadas por Pfafstetter (1957) o valor 1,10 (1,095) e com base em dados de vários pluviógrafos do Brasil, a Cetesb (1986) recomendou o valor 1,14. Segundo Froehlich (1993), o U.S. Weather Bureau indicou o valor de 1,13 e Kessler e Raad (1978) recomendaram o valor de 1,10. Weiss (1964), modelado em conceitos teóricos de probabilidade, estudou as relações entre precipitações médias obtidas por médias móveis e as precipitações obtidas em intervalos fixos, obtendo a relação de 1,143

Tabela 2 - Intensidade de chuva esperada $(\mathrm{mm} / \mathrm{h})$ para diferente duração e período de retorno para Videira, SC.

\begin{tabular}{|c|c|c|c|c|c|c|c|}
\hline \multirow{2}{*}{$\begin{array}{l}\text { Duração } \\
\text { (minutos) }\end{array}$} & \multicolumn{7}{|c|}{ Período de Retorno (anos) } \\
\hline & 100 & 50 & 25 & 20 & 10 & 5 & 2 \\
\hline 5 & 201,8 & 188,7 & 175,5 & 171,2 & 157,6 & 143,5 & 122,2 \\
\hline 10 & 164,4 & 151,7 & 139,0 & 134,9 & 121,9 & 108,3 & 87,8 \\
\hline 15 & 144,1 & 132,4 & 120,7 & 116,9 & 104,9 & 92,4 & 73,5 \\
\hline 20 & 132,5 & 121,7 & 110,8 & 107,2 & 96,1 & 84,5 & 66,9 \\
\hline 25 & 117,1 & 107,6 & 98,1 & 95,0 & 85,2 & 75,0 & 59,6 \\
\hline 30 & 109,9 & 100,7 & 91,5 & 88,5 & 79,0 & 69,2 & 54,3 \\
\hline 35 & 102,3 & 93,7 & 84,9 & 82,1 & 73,2 & 63,9 & 49,8 \\
\hline 40 & 92,9 & 85,2 & 77,4 & 74,9 & 67,0 & 58,8 & 46,3 \\
\hline 45 & 85,5 & 78,5 & 71,6 & 69,3 & 62,1 & 54,7 & 43,4 \\
\hline 50 & 79,3 & 73,0 & 66,6 & 64,6 & 58,0 & 51,2 & 41,0 \\
\hline 55 & 74,8 & 68,8 & 62,8 & 60,8 & 54,6 & 48,2 & 38,5 \\
\hline 60 & 70,5 & 64,8 & 59,1 & 57,2 & 51,4 & 45,3 & 36,1 \\
\hline 75 & 61,7 & 56,6 & 51,4 & 49,8 & 44,5 & 39,1 & 30,8 \\
\hline 90 & 56,1 & 51,3 & 46,5 & 44,9 & 39,9 & 34,8 & 27,0 \\
\hline 105 & 51,1 & 46,7 & 42,2 & 40,8 & 36,2 & 31,5 & 24,3 \\
\hline 120 & 47,2 & 43,1 & 39,0 & 37,6 & 33,4 & 28,9 & 22,2 \\
\hline 150 & 39,9 & 36,4 & 32,9 & 31,8 & 28,2 & 24,4 & 18,8 \\
\hline 180 & 34,0 & 31,1 & 28,2 & 27,3 & 24,3 & 21,2 & 16,5 \\
\hline 240 & 26,6 & 24,5 & 22,3 & 21,5 & 19,3 & 16,9 & 13,4 \\
\hline 300 & 22,5 & 20,7 & 18,8 & 18,2 & 16,3 & 14,3 & 11,3 \\
\hline 360 & 19,6 & 18,0 & 16,4 & 15,9 & 14,2 & 12,5 & 9,9 \\
\hline 420 & 17,3 & 15,9 & 14,5 & 14,1 & 12,7 & 11,2 & 8,9 \\
\hline 480 & 15,5 & 14,3 & 13,0 & 12,6 & 11,4 & 10,1 & 8,1 \\
\hline 600 & 12,4 & 11,5 & 10,6 & 10,3 & 9,3 & 8,3 & 6,8 \\
\hline 720 & 11,4 & 10,6 & 9,7 & 9,4 & 8,5 & 7,6 & 6,2 \\
\hline 840 & 10,4 & 9,6 & 8,8 & 8,5 & 7,7 & 6,9 & 5,6 \\
\hline 960 & 9,8 & 9,0 & 8,2 & 8,0 & 7,2 & 6,3 & 5,1 \\
\hline 1080 & 9,2 & 8,4 & 7,7 & 7,4 & 6,7 & 5,9 & 4,7 \\
\hline 1200 & 8,7 & 8,0 & 7,2 & 7,0 & 6,3 & 5,5 & 4,3 \\
\hline 1320 & 8,2 & 7,5 & 6,8 & 6,6 & 5,9 & 5,1 & 4,0 \\
\hline 1440 & 7,6 & 7,0 & 6,3 & 6,1 & 5,5 & 4,8 & 3,7 \\
\hline
\end{tabular}


Figura 1. Relação IDF de Videira, SC para chuvas com duração de 5 a 120 minutos.

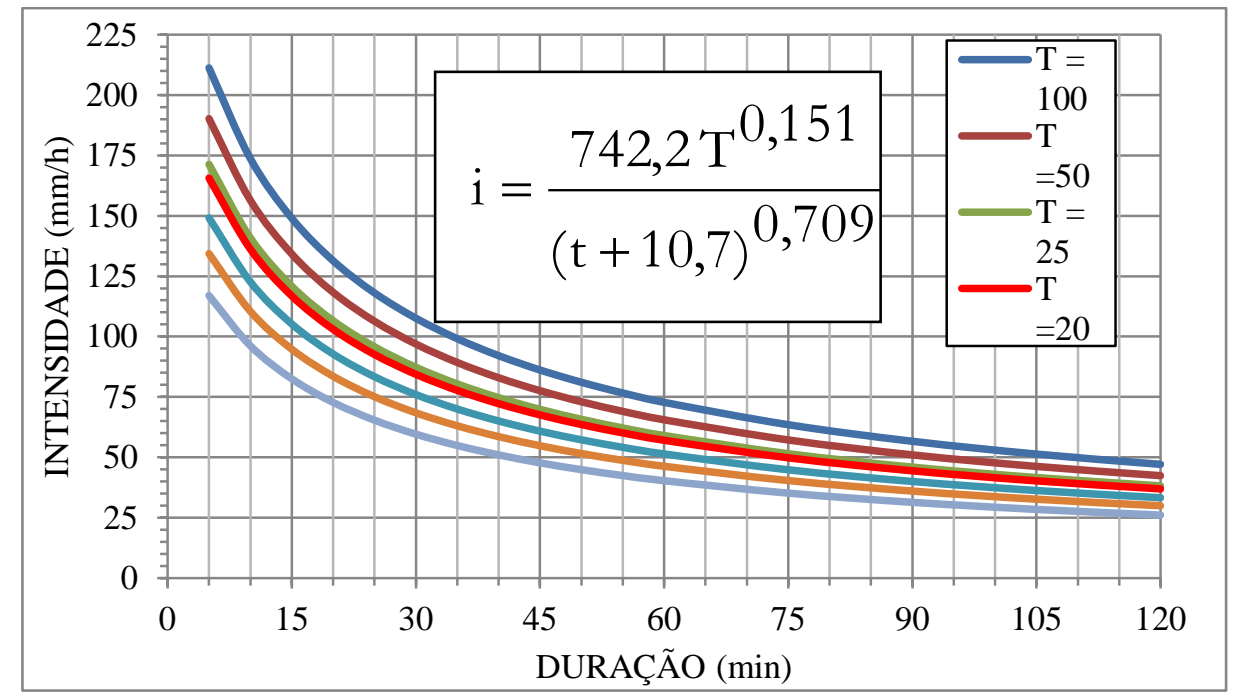

Figura 2. Relação IDF de Videira, SC para chuvas com duração de 120 até 1440 minutos.

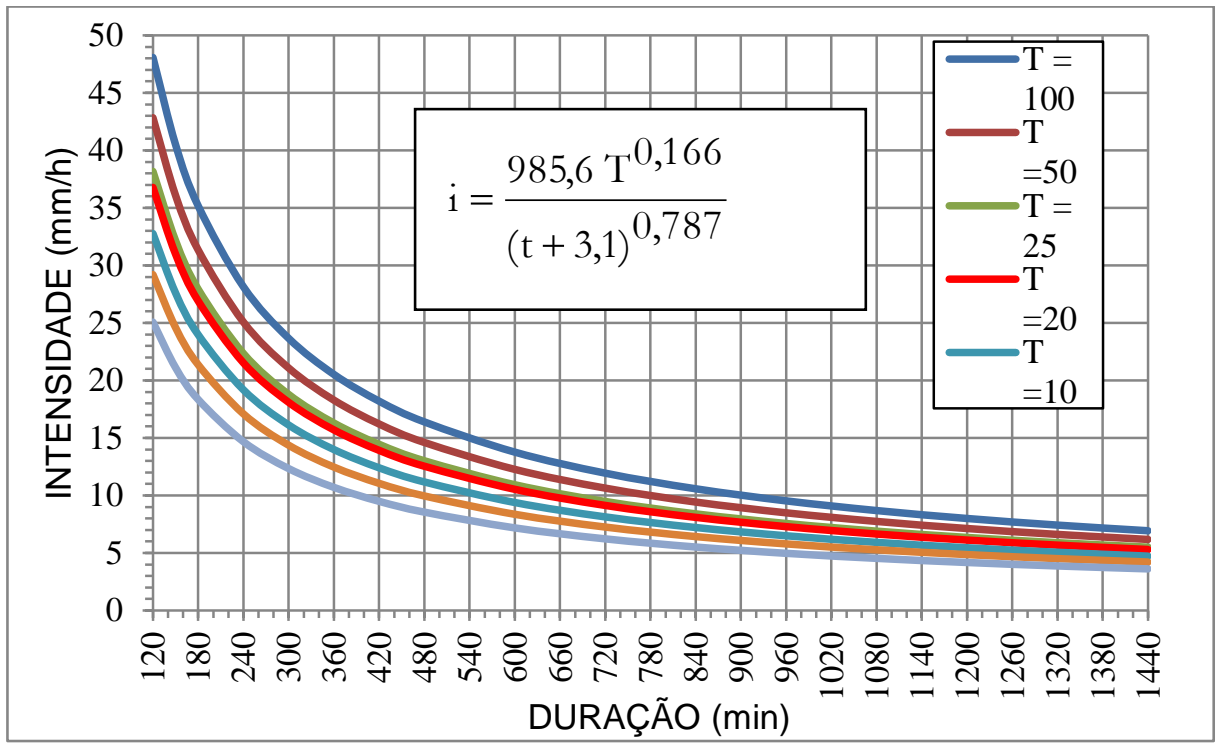

Nas estações meteorológicas a chuva de um dia é sempre medida em um horário fixo, normalmente as 9:00 horas horário de Brasília (12:00 horas de Greenwich). Neste estudo, foram analisadas as relações entre a chuva máxima em 24 horas e a chuva de um dia contabilizada nas diferentes horas do dia. Ocorre uma variação dessa relação conforme a hora da coleta da precipitação e também conforme o período de retorno considerado, variando de 1,08 a 1,20 (Figura 3). Considerando a média dos diversos períodos de retorno, observa-se que a relação varia de 1,10 para o horário das 11:00 h até 1,18 para o horário das 02:00 h. No horário da 09:00 h, quando é realizada a leitura dos pluviômetros nas estações meteorológicas do Inmet, a relação média é de 1,14, 
coincidindo com o valor indicado pela Cetesb (1986). Nas estações pluviométricas da Agência Nacional de Águas (ANA, 2009), a recomendação é realizar a leitura do pluviômetro as 07:00 horas, e para este horário a relação obtida de 1,16 foi ligeiramente superior. Considerando todas as durações, observou-se que a relação média variou de 1,15 para o período de retorno de 100 anos, a 1,13 para o período de retorno de 2 anos, com média de 1,14. Back (2015) analisando os dados da estação de Chapecó, observou que as relações entre a precipitação máxima de $24 \mathrm{~h}$ e a precipitação máxima de 1 dia variaram de 1,05 até 1,30, com média de 1,24. Observou também acentuada variação dessa relação nos diferentes horários, sendo os maiores valores observados para as 12:00 horas. O autor também concluiu que para a leitura as 09:00 horas essa relação média é de 1,24. Back et al. (2012) avaliaram as relações entre precipitações de diferentes durações para Santa Catarina e constataram que para a maioria das estações essa relação é ligeiramente superior a 1,14, sendo que para as estações de Itajaí, Florianópolis e Porto União essa relação média é de 1,30.

Figura 3 - Relação entre a precipitação máxima de 24 horas e a precipitação de 1 dia de Videira, SC conforme a hora da leitura da precipitação diária.

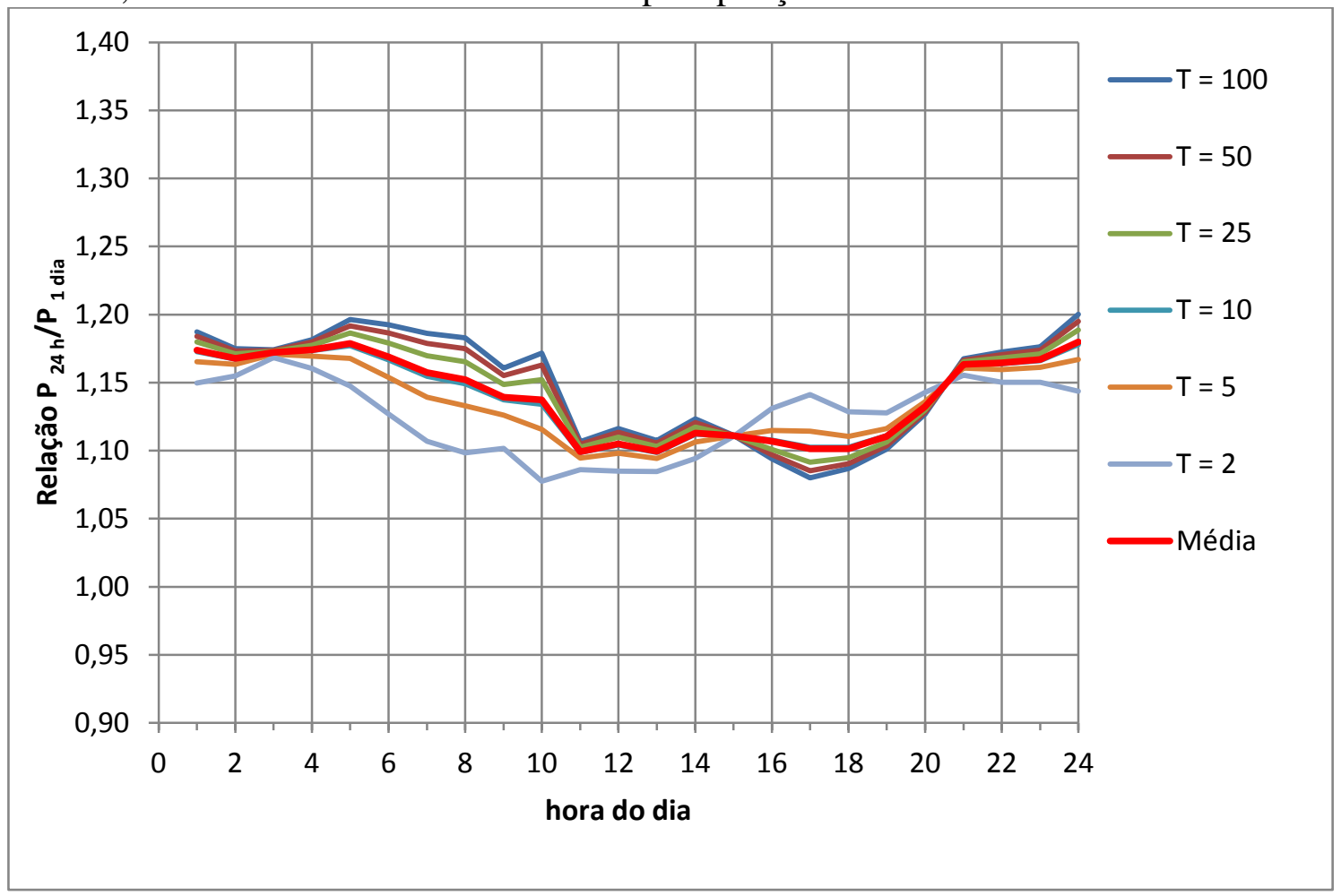

A relação entre a chuva máxima de 5 minutos e a chuva máxima em 30 minutos obtida neste estudo foi de 0,33, enquanto o trabalho da Cetesb (1986) indicava 0,34 (Tabela 3). Para as relações entre a chuva de 10, 15, 20 e 25 minutos com a chuva de 30 
minutos foram muito próximas as da Cetesb (1986), e em alguns casos foram iguais. Na relação entre a chuva máxima de 1 hora e a chuva de 24 horas foi obtida a média de 0,39, ligeiramente inferior a relação estabelecida pela Cetesb (1986) e é de 0,42. Como na estimativa de chuvas com duração inferior a 1 hora deve-se primeiro obter a chuva de 1 hora de duração, essa relação acaba afetando os valores da chuva de menor duração.

Tabela 3- Relações entre precipitações de diferentes durações de Videira, SC e valores médios publicados pela Cetesb (Cetesb, 1986).

\begin{tabular}{|c|c|c|c|c|c|c|c|c|c|}
\hline \multirow[b]{2}{*}{ Relações entre durações } & \multicolumn{8}{|c|}{$\mathrm{T}$ - Período de retorno (anos) } & \multirow[t]{2}{*}{ Cetesb } \\
\hline & 100 & 50 & 25 & 20 & 10 & 5 & 2 & média & \\
\hline $5 \mathrm{~min} / 30 \mathrm{~min}$ & 0,31 & 0,31 & 0,32 & 0,32 & 0,33 & 0,35 & 0,38 & 0,33 & 0,34 \\
\hline $10 \mathrm{~min} / 30 \mathrm{~min}$ & 0,50 & 0,50 & 0,51 & 0,51 & 0,51 & 0,52 & 0,54 & 0,51 & 0,54 \\
\hline $15 \mathrm{~min} / 30 \mathrm{~min}$ & 0,66 & 0,66 & 0,66 & 0,66 & 0,66 & 0,67 & 0,68 & 0,66 & 0,70 \\
\hline $20 \mathrm{~min} / 30 \mathrm{~min}$ & 0,80 & 0,81 & 0,81 & 0,81 & 0,81 & 0,81 & 0,82 & 0,81 & 0,81 \\
\hline $25 \mathrm{~min} / 30 \mathrm{~min}$ & 0,89 & 0,89 & 0,89 & 0,89 & 0,90 & 0,90 & 0,92 & 0,90 & 0,91 \\
\hline $30 \mathrm{~min} / 1 \mathrm{~h}$ & 0,78 & 0,78 & 0,77 & 0,77 & 0,77 & 0,76 & 0,75 & 0,77 & 0,74 \\
\hline $35 \mathrm{~min} / 1 \mathrm{~h}$ & 0,85 & 0,84 & 0,84 & 0,84 & 0,83 & 0,82 & 0,81 & 0,83 & - \\
\hline $40 \mathrm{~min} / 1 \mathrm{~h}$ & 0,88 & 0,88 & 0,87 & 0,87 & 0,87 & 0,87 & 0,86 & 0,87 & - \\
\hline $45 \mathrm{~min} / 1 \mathrm{~h}$ & 0,91 & 0,91 & 0,91 & 0,91 & 0,91 & 0,90 & 0,90 & 0,91 & - \\
\hline $50 \mathrm{~min} / 1 \mathrm{~h}$ & 0,94 & 0,94 & 0,94 & 0,94 & 0,94 & 0,94 & 0,95 & 0,94 & - \\
\hline $55 \mathrm{~min} / 1 \mathrm{~h}$ & 0,97 & 0,97 & 0,97 & 0,97 & 0,97 & 0,98 & 0,98 & 0,97 & - \\
\hline $1 \mathrm{~h} / 24 \mathrm{~h}$ & 0,39 & 0,39 & 0,39 & 0,39 & 0,39 & 0,39 & 0,40 & 0,39 & 0,42 \\
\hline $1,5 \mathrm{~h} / 24 \mathrm{~h}$ & 0,42 & 0,42 & 0,42 & 0,42 & 0,42 & 0,43 & 0,43 & 0,42 & - \\
\hline $2 \mathrm{~h} / 24 \mathrm{~h}$ & 0,46 & 0,46 & 0,46 & 0,46 & 0,46 & 0,45 & 0,45 & 0,46 & - \\
\hline $2,5 \mathrm{~h} / 24 \mathrm{~h}$ & 0,49 & 0,49 & 0,49 & 0,49 & 0,48 & 0,48 & 0,47 & 0,48 & - \\
\hline $3 \mathrm{~h} / 24 \mathrm{~h}$ & 0,52 & 0,51 & 0,51 & 0,51 & 0,51 & 0,50 & 0,49 & 0,51 & - \\
\hline $4 \mathrm{~h} / 24 \mathrm{~h}$ & 0,55 & 0,54 & 0,54 & 0,54 & 0,54 & 0,53 & 0,52 & 0,54 & - \\
\hline $5 \mathrm{~h} / 24 \mathrm{~h}$ & 0,56 & 0,56 & 0,56 & 0,56 & 0,56 & 0,55 & 0,55 & 0,56 & - \\
\hline $6 \mathrm{~h} / 24 \mathrm{~h}$ & 0,58 & 0,58 & 0,59 & 0,59 & 0,59 & 0,59 & 0,60 & 0,59 & 0,72 \\
\hline $7 \mathrm{~h} / 24 \mathrm{~h}$ & 0,62 & 0,62 & 0,62 & 0,62 & 0,62 & 0,62 & 0,63 & 0,62 & - \\
\hline $8 \mathrm{~h} / 24 \mathrm{~h}$ & 0,64 & 0,64 & 0,65 & 0,65 & 0,65 & 0,65 & 0,66 & 0,65 & - \\
\hline $10 \mathrm{~h} / 24 \mathrm{~h}$ & 0,66 & 0,67 & 0,67 & 0,67 & 0,67 & 0,68 & 0,69 & 0,67 & 0,82 \\
\hline $12 \mathrm{~h} / 24 \mathrm{~h}$ & 0,68 & 0,68 & 0,69 & 0,69 & 0,69 & 0,70 & 0,72 & 0,69 & 0,85 \\
\hline $14 \mathrm{~h} / 24 \mathrm{~h}$ & 0,68 & 0,69 & 0,70 & 0,70 & 0,71 & 0,73 & 0,76 & 0,71 & - \\
\hline $16 \mathrm{~h} / 24 \mathrm{~h}$ & 0,75 & 0,76 & 0,77 & 0,77 & 0,78 & 0,79 & 0,83 & 0,78 & - \\
\hline $18 \mathrm{~h} / 24 \mathrm{~h}$ & 0,80 & 0,80 & 0,81 & 0,81 & 0,82 & 0,84 & 0,87 & 0,82 & - \\
\hline $20 \mathrm{~h} / 24 \mathrm{~h}$ & 0,85 & 0,86 & 0,86 & 0,87 & 0,87 & 0,89 & 0,91 & 0,87 & - \\
\hline $22 \mathrm{~h} / 24 \mathrm{~h}$ & 0,90 & 0,90 & 0,91 & 0,91 & 0,92 & 0,92 & 0,94 & 0,91 & - \\
\hline $24 \mathrm{~h} / 1 \mathrm{dia}$ & 1,15 & 1,15 & 1,14 & 1,14 & 1,14 & 1,14 & 1,13 & 1,14 & 1,14 \\
\hline
\end{tabular}

Observa-se assim que o uso da relação da Cetesb implica em ligeira superestimativa dos valores de chuva quando comparada com os valores observados em 
Videira. Lobo e Magni (1987), com base em 11 estações pluviográficas do Estado de São Paulo, encontraram essas relações com valor de 0,51. Para as durações entre as chuvas de 6, 10, 12 horas e a chuva de 24 horas também foram encontradas relações ligeiramente inferiores as estabelecidas pela Cetesb. Esse comportamento já foi observado por Back (2012) nas diversas estações pluviográficas do Estado de Santa Catarina, sendo as diferençam mais acentuadas para as estações de Florianópolis, Laguna e Itajaí.

\section{Conclusões}

Este trabalho permitiu obter as seguintes conclusões para os dados de precipitação intensa de Videira:

$\checkmark$ A distribuição de Gumbel-Chow se mostrou adequada para a estimativa das chuvas máximas anuais com duração 5 min até 1440 minutos;

$\checkmark$ As equações de chuvas intensas ajustadas fornecem estimativas de valores muito próximos dos valores observados;

$\checkmark$ Para a chuva coletada às nove horas a relação entre a chuva máxima em 24 horas e a chuva máxima de um dia é de 1,14.

$\checkmark$ As relações entre chuvas de diferentes durações obtidas para Videira apresentaram valores ligeiramente inferiores aos valores médios recomendados para o Brasil por Cetesb (1986).

\section{Agradecimentos}

Os autores agradecem o apoio financeiro do Conselho Nacional de Desenvolvimento Científico e Tecnológico - CNPq-Brasil.

\section{Referências}

Agência Nacional de Águas (ANA). Inventário das estações pluviométricas. Agência Nacional de Águas. 2ed. Brasília: SGH, 2009, 332p.

BACK, Á.J. Relações IDF de Chapecó-SC atualizadas com dados de 1976 a 2014. In: SIMPOSIO BRASILEIRO DE RECUROSOS HIDRICOS, 21., 2015, Brasília, Anais...Porto Alegre. ABRH, 2015.

BACK, Á.J. Chuvas intensas e chuva para dimensionamento de estruturas de drenagem para o Estado de Santa Catarina (Com programa HidroChuSC para cálculos). Florianópolis: Epagri, 193p. 2013.

BACK, Á.J.; OLIVERIA, J.L.R.; HENN, A. Relações entre precipitações de diferentes durações para desagregação da chuva diária de Santa Caarina. Revista Brasileira de Engenharia Agrícola e Ambiental, v.16, n.4, p., 2012, 
BERTONI, J. C.; TUCCI, C. E. Precipitação. In: TUCCI, C. E. Hidrologia: Ciência e Aplicação. Porto Alegre. Editora da Universidade. cap.5. p.177-241. 1993.

CETESB. Drenagem urbana - manual de projeto. $3^{\text {a }}$ ed. São Paulo. 464 p. 1986.

CHOW, V. Handbook of applied hydrology. New York: McGraw-Hill, 1418 p. 1964.

CLARKE, R.T. Statstical Modelling in hydrology. John Wiley \& Sons, England. 1994.

CLARKE, R. T. Fitting and testing the significance of linear trends in Gumbel distributed data. Hydrology and Earth System Sciences, Katlenburg-Lindau, Alemanha, v.6, n. 1, p.17-24. Feb, 2002.

FROEHLICH, D. C. Short-duration-rainfall intensity equations for drainage design. Journal of Irrigation and Drainage Engineering, v.119, p.814-828, 1993.

GENOVEZ, A. M.; PEGORARO, R. C. F. Análise e avaliação de equações de chuvas intensas generalizadas. In: Congresso Latinoamericano de Hidráulica 18, 1998, Oaxaca. Anais... Oaxaca: IAHR, v.1, 1998, p.369-379.

GENOVEZ, A. M.; ZUFFO, A. C. Chuvas intensas no Estado de São Paulo: estudos existentes e análise comparativa. Revista Brasileira de Recursos Hídricos, v.5, p.45$58,2000$.

KESSLER, J.; RAAD, S. J. Análise de dados pluviométricos. In: International Institute for Land Reclamation and Improvement. Principios y aplicaciones del drenaje. Wageningen: ILRI. v.3, 1978, p.16-57, Publication 16.

LOBO, G. A.; MAGNI, N. L. G. Estudo sobre proporções entre chuvas intensas de diferentes durações. In: VII Simpósio Brasileiro de Hidrologia e Recursos Hídricos. Anais... Salvador: ABRH, v.2, 1987, p.103-114.

NEGHETTINI, M.; PINTO, E.J. A. Hidrologia estatística. Belo Horizonte, CPRM, 552p. 2013.

OCCHIPINTI, A. G.; SANTOS, P. M. Relações entre as precipitações máximas de um dia e de 24 horas na cidade de São Paulo. São Paulo: IAU/USP, 1966.10p

PFAFSTETTER, O. Chuvas intensas no Brasil: Relação entre precipitação, duração e freqüência de chuvas em 98 postos com pluviógrafos. Rio de Janeiro: DNOCS, 419p. 1957.

TOMAZ, P. Cálculos hidrológicos e hidráulicos para obras municipais. São Paulo. Navegar Editora. 475p.. 2002.

TORRICO, J. J. T. Práticas hidrológicas. Rio de Janeiro: Transcon, 1975. 119p.

WEISS, L. L. Ratio of true to fixed-interval maximum rainfall. Journal of Hydraulics Division, v.90, p.77-82, 1964. 